\title{
ÍNDICE DE NAHUATILISMOS EN EL ESPAÑOL DE LA FRONTERA MEXICANA CON BELICE
}

\author{
Raúl Arístides Pérez Aguilar \\ Universidad de Quintana Roo \\ rauperez@uqroo.mx
}

\begin{abstract}
Resumen
En este artículo se analiza la presencia de las voces nahuas en la frontera mexicana con Belice, las cuales fueron obtenidas mediante la aplicación de un cuestionario que dio como resultado un valioso corpus lingüistico que permite analizar el uso real de estas voces (léxico activo), así como establecer su nómina pasiva, las circunstancias de su uso y de los sinónimos con los que entran en competencia, su significación sociolingüística de acuerdo con los factores de edad, sexo y grado de escolaridad, y la norma lingüística de cada grupo sociocultural y la norma general de la ciudad.
\end{abstract}

PALABRAS CLAVE: Nahuatlismos, Belice, Chetumal, Léxico.

\begin{abstract}
This article analyzes the presence of Nahua ter$\mathrm{ms}$ in the Mexico-Belize border area. These terms were gathered through a questionnaire, resulting in a valuable linguistic corpus which allows us to analyze the real use of these terms (active lexicon), as well as their passive use, the circumstances of use and of the synonyms with which they compete, their sociolinguistic meaning according to age, sex and educational levels, including the linguistic habits of each sociocultural group and the more general use of these terms in the city of Chetumal.
\end{abstract}

KEY WORDS: Nahua terms, Belize, Chetumal, Lexicon.

A Juan M. Lope Blanch (In memoriam).

\section{Introducción}

Una de las recomendaciones que de manera constante hacía Juan M. Lope Blanch en sus estudios lexicográficos era que debería investigarse la vitalidad y el prestigio de los indigenismos en las distintas generaciones de hablantes y en los diversos niveles socioculturales, ${ }^{1}$ con objeto de no cometer peligrosas hipótesis sociolingüísticas. A esta invitación agregaba que también estos trabajos de investigación deberían ser realizados en varias zonas del país - y no solamente en la ciudad de México- con idéntico objetivo.

Ante la diversidad léxica mexicana que conocemos gracias al Atlas Lingüistico de México en sus tomos 5 y 6 (sea por ejemplo la designación de bebé —mapa 945-: chiquillo, nené, bebé, conete, pichito, chilpayate, niño pequeño, niño de brazos, nene...), es evidente

1 Véase su trabajo "Indigenismos americanos en la norma lingüística culta de México" en Investigaciones sobre dialectología mexicana, México, UNAM, 1987, p. 147-160, en especial las páginas 157 y 158, en el que presenta el caso de la voz tianguis. 
que la sugerencia de Lope Blanch cobra particular importancia, sobre todo en estos tiempos de globalización y cambios profundos en las comunidades lingüísticas.

En una región como en la que se encuentra situada la ciudad de Chetumal -frontera de México con Centroamérica y el Caribe-, en donde difícilmente no podrían hallarse en los estilos coloquial y aun en el formal oral la presencia considerable de indigenismos léxicos de diverso origen - desde los que poseen un hondo matiz náhuatl y maya hasta los discutidos antillanismos -2 , la realización de un estudio que manifieste la vitalidad de éstos se hace necesaria.

Ante la particular situación que ofrece el habla de esta comunidad, pretendí - a través de la aplicación de un cuestionario - saber qué tan vivas se hallan estas voces indígenas -en especial los nahuatlismos - en la sociedad chetumaleña.

Al no existir estudio alguno acerca de este tipo de léxico en la norma de la ciudad, el trabajo entonces consistió en echar mano de la propia experiencia de vida ${ }^{3}$ y de los varios estudios lexicológicos que se han llevado a cabo tanto en México como en otros países de América $^{4}$ para la elaborar la nómina que habría de formar el cuestionario.

Ya en otro lugar ${ }^{5}$ me he referido a la situación que presentan en el habla chetumaleña 43 mayismos; sin embargo, no son sólo éstos - que por su presencia constante en cualquier situación y por su raigambre étnica podría pensarse que fueran los más abundantes- sino también las voces procedentes de otras lenguas de América - en especial la náhuatl del altiplano de México - las que en conjunción caracterizan el habla de la comunidad.

De este modo, la confluencia de estas palabras con sus sinónimos hispánicos dan muestra de la riqueza léxica que posee esta sociedad en la que se hallan mezcladas varias culturas - la caribe, la criolla de Belice, la maya y la mexicana- y sus costumbres lingüísticas.

\section{Propósitos y metodología}

Los principales objetivos de este trabajo son: 1. Registrar la vigencia real de los nahuatlismos (léxico activo) en el español de Chetumal, 2. Analizar la posible significación sociolingüística de este léxico de acuerdo con las variables de edad, escolaridad y sexo, 3. Establecer las circunstancias de uso de estas voces y los sinónimos con los que entran en competencia,4. Establecer la nómina pasiva de los nahuatlismos en la norma de cada nivel sociocultural y en la norma general de la ciudad.

2 La discusión acerca del origen de varios antillanismos rebasa con mucho mis pretensiones. Sin embargo, en cuanto a su vitalidad, puede verse mi trabajo "Antillanismos en el Caribe mexicano" en Cuicuilco, vol. 9, núm. 26, México, 2002, págs. 255-269.

3 La propia condición de ser nativo de Chetumal me ayudó sobremanera en la elaboración de la nómina que estructura la encuesta aplicada. Pienso, al igual que Montes (1982), que en general el encuestador nativo con suficiente preparación previa parece preferible sobre el foráneo pues conoce mejor la vida de su región y no deja escapar hechos importantes, a pesar de que presenta ciertas desventajas sobre todo en la percepción de fenómenos fonéticos y gramaticales que están dentro de sus hábitos lingüísticos (p. 73 y siguientes.).

4 Véanse los trabajos de Lope Blanch (1979), Alba (1976), Henríquez Ureña (1935, 1938), Morinigo (1985), Vaquero (1986), y Santamaría (1984) entre muchos otros que abordan el tema de los indigenismos en el español americano.

5 Véase mi artículo"Vitalidad y significación sociolingüística de los mayismos en el español de Chetumal" en Lingüística mexicana, Vol. I, núm. 2 México, 2000, p. 181-195. 
El proceso para la obtención de los datos consistió en la aplicación de un cuestionario estructurado por 259 nahuatlismos ${ }^{6}$.

Hecha la nómina de voces, se aplicó a cada una esta serie de preguntas con diversos objetivos:

1. ¿Conoce usted esta palabra? Sí. No

Si el informante respondía sí, entonces...

2. ¿Con qué sentido la conoce? (y se anotaba el significado).

3. ¿La usa? Sí No.

Si el informante respondía sí, entonces...

4. ¿En qué circunstancia la usa? (y se anotaba el contexto). Finalmente...

5. ¿Con qué otro nombre conoce el objeto, la fruta, la cualidad, etc?

El cuestionario fue contestado en presencia del encuestador quien anotaba las respuestas obtenidas para cada cuestión, las observaciones que hacía el encuestado acerca del significado y del contexto de uso de la voz así como los datos generales de éste.

Los informantes que conformaron la muestra seleccionada fueron hombres y mujeres hispanohablantes nacidos en Chetumal o con más de 10 años de residencia en la ciudad, distribuidos en tres niveles de escolaridad: bajo - hasta $6^{\circ}$ de primaria-, medio - hasta con el bachillerato concluido- ${ }^{7}$ y alto -estudios posteriores al bachillerato; y grupo generacional: jóvenes de 20 a 30 años (grupo I), maduros -hasta los 50 años (grupo II), y mayores de 50 años (grupo III). Fueron encuestados así 300 individuos ( 150 hombres y 150 mujeres, 100 del nivel bajo, 100 del medio y 100 del alto; 100 jóvenes, 100 maduros y 100 ancianos).

\section{Resultados generales}

Después de tabular los datos, de las 259 unidades léxicas que componen el cuestionario, 22 resultaron ser completamente desconocidas por todos los informantes. ${ }^{8}$ Las palabras desconocidas fueron obviamente desechadas, y de las 237 restantes, $109^{9}(42.08 \%)$ estructuran en conjunto la nómina pasiva del léxico náhuatl en el español de Chetumal, ya que para considerar un elemento lingüístico como perteneciente a este léxico se requirió que éste fuera conocido por más del $50 \%$ de los hablantes

De lo anterior se desprende que de la nómina aplicada, 150 nahuatlismos no pertenecen al léxico pasivo ( 22 totalmente desconocidas más 128 que resulta de la resta de 237 menos 109 ), es decir, estas voces no son conocidas ni por el $50 \%$ de los encuestados.

6 La nómina la tomé integramente de la presentada por Lope Blanch (1979).

7 Se tomó en algunos informantes una carrera comercial corta como equivalente al bachillerato.

8 Éstas corresponden solamente a los nahuatlismos ahuacle, áxcale, ayacahuite, camichín, cuescomate,cuitla, chalchihuil, chomite, huehuenche, nautle, nexcomil, quiote, tayacán, tecotehue, tenamascle, tecal, tlacuil, xolosóchil, yagual, zacahuiste, zacamiche y zontle. Las voces que aparecen en negritas también las registra como inusuales Lope Blanch (1979) quien documenta otras más, mientras que Lara (1996) sólo asienta como usual - al menos en el altiplano de México- ayacahuite.

9 Que corresponden a 77 lexemas. 
De manera general, las $109^{10}$ unidades léxicas estudiadas pertenecen a la nómina pasiva de los nahuatlismos, pero dentro de ésta existen diferencias. Es decir, $38^{11}$ poseen la característica de ser absolutamente conocidas, $26^{12}$ exhiben un porcentaje que oscila entre el 81 y el $99 \%$ de conocimiento, mientras que las $45^{13}$ restantes sitúan su porcentaje entre el 51 y el $80 \%$.

\section{Resultados según variables (léxico pasivo)}

Cabe hacer mención que de toda esta nómina pasiva, las palabras que presentaron las diferencias más significativas fueron nagual desconocida por el sector inculto y por el grupo generacional joven, y náhuatl que no fue identificada por ninguno de los miembros del grupo menos escolarizado. Estas palabras por lo tanto, resultan ser caracterizadoras sobre todo de los diversos sociolectos de la ciudad y de los distintos grupos generacionales.

Ahora bien, para que una unidad léxica fuera considerada como caracterizadora de algún grupo social, generacional o de sexo, se consideró que debía presentar una diferencia en el conocimiento o en el uso de más del $25 \%$ con respecto a los otros sectores.

Así por ejemplo resultaron más propias de los hombres las voces nauyaca, achichincle y chicloso; de las mujeres: cenzontle y nagual; del sector de personas maduras: apapachar,

10 En esta nómina el primer porcentaje — dado en números enteros - se refiere al léxico pasivo y el segundo al activo: Achichincle $(56,29)$, achiote $(100,76)$, aguacate $(100,100)$, ahulado $(73,50)$, apapachar $(88,87)$, atole $(100,100)$, cacahuate $(100,100)$, cacao $(100,63)$ camote $(100,100)$, cempasúchil $(60,44)$, cenzontle $(76,43)$, comal $(100,100)$, coyote $(100,48)$, cuate $(100,65)$, chachalaca $(76,78)$, chamaco $(100,73)$, chapopote $(86,65)$, chapulin $(83,36)$, chaquiste $(83,44)$, chayote $(100,100)$, chicle $(100,100)$, chiclero $(96,48)$, chicloso $(83,37)$, chichi $(53,37)$, chicozapote $(100,46)$, chihuahua $(100,100)$, chilaquiles $(100,100)$, chile $(100,100)$, chilmole (chirmole) $(100,76)$, chilpayate $(83,48)$, chilpachole $(56,47)$, chinampa $(60,16)$, chipote $(76,47)$, chipotle $(73$, $63)$, chocolate $(100,100)$, chocolateria $(70,23)$, chocolatero $(66,30)$, enchapopotar $(53,25)$, enchilada $(100,100)$, enchilarse $(100,100)$, ejote $(63,36)$, elote $(100,100)$, enjitomatar $(60,38)$, epazote $(100,73)$, escuincle $(90,62)$, guacamole $(100,80)$, guachinango $(96,48)$, guajolote $(90,48)$, huacal $(83,72)$, huapango $(63,36)$, huipil $(93,57)$, hule $(100,77)$, jacal $(90,37)$, jicama $(100,83)$, jicara $(90,40)$, jitomate $(100,63)$, machote $(66,40)$, malinchismo $(60,55)$, malinchista $(56,64)$, mapache $(86,57)$, matatena $(63,36)$, mayate $(66,40)$, mecate $(76,47)$, memela $(60,33)$, metate $(63,31)$, mezcal $(96,37)$, milpa $(100,50)$, mitotero $(50,26)$, molcajete $(76,86)$, mole $(100,100)$, nagual $(66,10)$, náhuatl $(63,26)$, nanche $(66,75)$, nauyaca $(63,36)$, nixtamal $(73,36)$, nopal $(100,66)$, nopalera $(73,27)$, paliacate $(93,46)$, papalote $(93,60)$, pepenador $(70,28)$, pepenar $(63,31)$, petaca $(83,40)$, petacón $(60,33)$, petate $(73,27)$, petatearse $(76,47)$, pinole $(56,41)$, popote $(100,80)$, pozole $(100,100)$, pulque $(96$, $31)$, pulqueria $(83,20)$, pulquero $(73,22)$, quetzal $(80,29)$, tamal $(100,100)$, tecolote $(93,32)$, tejocote $(63,42)$, tepache $(100,50)$, tequila $(100,100)$, tianguis $(83,52)$, tlacuache $(66,30)$, tlapalería $(90,59)$, tocayo $(83,68)$, toloache $(56,29)$, tomate $(100,100)$, totopo $(66,35)$, tuza $(66,35)$, zacatal $(66,35)$, zacate $(100,100)$, zapote (100, 76), zopilote $(100,100)$.

11 Achiote, aguacate, atole, cacahuate, cacao, camote, comal, coyote, cuate, chamaco, chayote, chicle, chicozapote, chihuahua, chilaquiles, chile, enchilada, enchilarse, chocolate, elote, epazote, guacamole, hule, jicama, jitomate, milpa, mole, chilmole (chirmole), nopal, popote, pozole, tamal, tepache, tequila, tomate, zacate, zapote, zopilote.

12 Ahulado, apapachar, chapopote, chapulin, chaquiste, chiclero, chicloso, chilpayate, escuincle, guachinango, guajolote, huacal, huipil, jacal, jicara, mapache, mezcal, paliacate, papalote, petaca, pulque, pulqueria, tecolote, tianguis, tlapaleria, tocayo.

13 Achichincle, cempasúchil, cenzontle, chachalaca, enchapopotar, chichi, chilpachole, chinampa, chipote, chipotle, chocolateria, chocolatero, ejote, huapango, enjitomatar, machote, malinchismo, malinchista, matatena, mayate, mecate, memela, metate, mitotero, molcajete, nagual, náhuatl, nanche, nauyaca, nixtamal, nopalera, pepenar, pepenador, petacón, petate, petatearse, pinole, pulquero, quetzal, tejocote, tlacttache, toloache, totopo, tuza, zacatal. 
malinchista, tlacuache y achichincle; de la gente de mayor edad: chilpachole, ejote, malinchismo, matatena, mitotero; del sector culto: ejote, achichincle, chilpachole, mitotero, malinchismo, malinchista, petacón y machote.

En adición a esto la observación de los siguientes cuadros proporcionará algunos resultados dignos de considerarse en el análisis de la nómina.

\section{Cuadro I}

Porcentaje de nahuatlismos (léxico pasivo) según escolaridad

\begin{tabular}{|c|c|c|c|}
\hline & Bajo \% & Medio \% & Alto \% \\
\hline Nahuatlismos & 72.60 & $\mathbf{8 1 . 5 5}$ & 92.84 \\
\hline
\end{tabular}

Del cuadro anterior se desprende - como era de esperarse- que el grupo con escolaridad superior es el que conoce un mayor número de nahuatlismos, y el más precario resulta ser el sector inculto. El porcentaje de conocimiento de estas voces en el sector medio se halla más cercano al del nivel bajo que al del superior, por lo que se puede afirmar que no solamente el sector menos escolarizado es el más rezagado en el conocimiento de estas voces amerindias.

\section{Cuadro II}

Porcentaje de nahuatlismos (léxico pasivo) según edad

\begin{tabular}{|c|c|c|c|}
\hline & I \% & II \% & III \% \\
\hline Nahuatlismos & $\mathbf{7 5 . 9 0}$ & $\mathbf{8 1 . 8 5}$ & $\mathbf{9 4 . 8 8}$ \\
\hline
\end{tabular}

La observación del cuadro II evidencia marcadas diferencias entre los sectores generacionales que conforman la muestra. Como puede apreciarse, la mayor riqueza léxica radica en las personas de más de 50 años, y la menor en la gente de menos de 30 , lo que indica que existe un desconocimiento lexical patente en estos jóvenes que se observa en el rango entre ambos sectores (18.98\%). Por su parte, el sector de la gente madura posee un conocimiento de estos indigenismos más cercano al que tienen los jóvenes que al que exhiben los de mayor edad, situación que permite ver por un lado - una vez más- que el grupo que más conserva estas voces es el de las personas de edad avanzada, y que hace suponer, por el otro, que cada día el número de nahuatlismos conocidos será menor.

Cuadro III

Porcentaje de nahuatlismos (léxico pasivo) según sexo

\begin{tabular}{|c|c|c|}
\hline & Hombres \% & Mujeres \% \\
\hline Nahuatlismos & $\mathbf{8 1 . 7 7}$ & $\mathbf{8 2 . 0 7}$ \\
\hline
\end{tabular}

La distribución de los indigenismos de la nómina pasiva en los grupos sexuales ofrece menos disparidad. De manera general, el conocimiento de las voces es un poco mayor en el sexo femenino que en el masculino.

Establecida de este modo la nómina pasiva en cada uno de los factores sociolingüísticos y en la norma general de la ciudad, es necesario conocer el porcentaje del uso real de los 
nahuatlismos; es decir, el léxico activo. Como es lógico suponer que el hablante sólo usa las palabras que conoce, el porcentaje de uso de cada unidad léxica fue calculado sobre el total de informantes que dijeron conocerla y no sobre la totalidad de la muestra. De lo anterior se desprende que:

1. El porcentaje de uso sea menor que el del conocimiento.

2. Ambos porcentajes coincidan (el caso de tamal, conocida y usada por todos los informantes).

3. El porcentaje de uso sea mayor que el del conocimiento

Es necesario anotar que de las 109 unidades léxicas estudiadas, sólo 21 de ellas presentan un $100 \%$ de uso real ${ }^{14}$; oscilan entre el $50 \%$ y el $99 \%$ un total de 32 voces, ${ }^{15}$. Las 56 unidades léxicas restantes son usadas por menos del $50 \%$ de la muestra. ${ }^{16}$

\section{Resultados según variables (léxico activo)}

Siguiendo el mismo procedimiento utilizado en la nómina pasiva, se requirió que cada unidad léxica presentara una diferencia del $25 \%$ de uso entre los diversos sectores sociales, generacionales y de sexo para que fuera considerada como caracterizadora.

Resultaron así ser más usuales los nahuatlismos 1. apapachar, 2. cenzontle, 3. chilpachole, 4. malinchista, 5. nauyaca, 6. tlacuache, 7. chocolate en el sector generacional II (1,4 y 6), en las mujeres (2) y en los varones (5 y 7), y en los sectores inculto y alto (3). Las 102 unidades léxicas nahuas restantes corresponden a la norma general de la ciudad.

Una vez caracterizados los grupos de la muestra según el uso de las unidades léxicas estudiadas, veamos más en detalle los resultados de estas observaciones:

\section{Cuadro IV}

Porcentaje de nahuatlismos (léxico activo) según escolaridad

\begin{tabular}{|c|c|c|c|}
\hline & Bajo \% & Medio \% & Alto \% \\
\hline Nahuatlismos & 29.57 & 33.94 & 31.26 \\
\hline
\end{tabular}

La observación detenida de los resultados del cuadro IV, permite ver que es el sociolecto medio el que señorea. Prácticamente el uso de los nahuatlismos en este sector es mayor que en los otros dos. La posición intermedia de este grupo permite realizar el contraste de los datos

El menor uso de indigenismos en el nivel inculto es explicable por su propia condición de ignorancia, sin embargo resulta extraño que el sector más escolarizado tenga un comportamiento débil en cuanto al uso de estas voces indígenas, ¿acaso su propia cultura

\footnotetext{
14 Aguacate, atole, cacahuate, camote, comal, chayote, chihuahua, chilaquiles, chile, enchilada, enchilarse, chocolate, elote, mole, nopal, pozole, tamal, tequila, tomate, zacate y zopilote.

15 Achiote, ahulado, apapachar, cacao, cuate, chachalaca, chamaco, chapopote, chipotle, epazote, escuincle, guacamole, huacal, huipil, hule, jicama, jitomate, malinchismo, malinchista, mapache, milpa, molcajete, chilmole, nanche, nopal, papalote, popote, tepache, tianguis, tlapaleria, tocayo, zapote,

16 Achichincle, cempasúchil, cenzontle, coyote, enchapopotar; chapulin, chaquiste, chiclero, chicloso, chichi, chicozapote, chilpachole, chilpayate, chinampa, chipote, chocolateria, chocolatero, ejote, guachinango, guajolote, huapango, jacal, jicara, enjitomatar, machote, matatena, mayate, mecate, memela, metate, mezcal, mitotero, nagual, náhuatl, nauyaca, nixtamal, nopalera, paliacate, pepenar, pepenador, petaca, petacón, petate, petatearse, pinole, pulque, pulqueria, pulquero, quetzal, tecolote, tejocote, tlacuache, toloache, totopo, tuza, zacatal.
} 
lo ha proveído de sinónimos abundantes que han hecho que no use varios vocablos, o es la condición privilegiada de ser culto y debido a ello negarse a utilizar voces provenientes de lenguas con menos prestigio que la española? La diferencia entre el conocimiento y el uso de nahuatlismos en este sector es enorme $(61.58 \%)$, situación que podría ser explicada mediante un estudio de conciencia sociolingüística. Los otros dos sectores socioculturales ofrecen menos discrepancias entre el léxico pasivo y el activo: $43.03 \%$ el nivel inculto, y $47.61 \%$ el medio.

De esto se desprende que el grupo menos escolarizado, a pesar de que posea un menor conocimiento de los nahuatlismos, en su comunicación cotidiana los usa más; estas voces forman una especie de léxico básico, léxico con una mayor estabilidad estadística al que los hablantes recurren a menudo para estructurar sus mensajes, independientemente del tema de la conversación.

Lo contrario suele ocurrir en el sector alto en el que la amplitud de la nómina indígena que forma parte del léxicón mental de los hablantes de este sociolecto no suele realizarse siempre y cotidianamente, pues ha sido obstaculizada con sinónimos provenientes de lecturas, viajes, empleos, etc. Es decir, a un mayor conocimiento de voces no indígenas corresponde un menor uso real de cada nahuatlismo, pues la sinonimia auxilia al hablante en el momento de conversar.

Es de esperarse que en el sector medio confluyan ambas situaciones lingüísticas —ni un léxico indígena tan restringido como el del nivel inculto, ni un lexicón no indígena tan amplio con el del grupo más escolarizado - y por ello exhiba un mayor porcentaje de léxico activo indígena.

\section{Cuadro V}

Porcentaje de nahuatlismos (léxico activo) según edad

\begin{tabular}{|c|c|c|c|}
\hline & I \% & II \% & III \% \\
\hline Nahuatlismos & 31.16 & 35.66 & 28.15 \\
\hline
\end{tabular}

Los resultados que arroja el cuadro $\mathrm{V}$ muestran que el mayor uso de los nahuatlismos radica en el grupo de personas entre 30 y 50 años, siendo el sector de mayor edad el que menos echa mano de tales vocablos, lo que indica una reactivación en el uso de este léxico si se toma en cuenta que el sector II no es el que posee la mayor nómina pasiva de los tres niveles ( $81.85 \%$ frente al $94.88 \%$ del grupo III). (Véase cuadro II). Este fenómeno de reactivación es sólo relativo si se piensa en la gran cantidad de palabras que reposan en la mente de los hablantes de mayor edad y que está, desgraciadamente, destinado a desaparecer. La lógica suposición de que a mayor edad corresponde una mayor riqueza léxica activa es también sumamente relativa si se toma en cuenta lo restringido del mundo de los ancianos, sobre todo los incultos (falta de empleo y lecturas, soledad, etc.,) que hace que estas voces hayan caído en el olvido y por lo tanto en el desuso. Por otro lado, la mayor movilidad social del nivel II y su comunicación en diversas actividades diarias lo provee de circunstancias en las que necesitan aflorar estos indigenismos. De ahí que este sector manifieste la mayor vitalidad de éstos.

El grupo de jóvenes, considerado como el menos conservador y sí como el más innovador, también muestra un uso reducido de estas voces indígenas. La influencia de la televisión y de la radio, y las circunstancias de una vida muy distinta a la de las personas de mayor 
edad han originado que muchas voces como matatena, chaquiste, tlacuache y tule tengan un uso incipiente en la conversación cotidiana de este grupo social.

\section{Cuadro VI}

Porcentaje de nahuatlismos (léxico activo) según sexo

\begin{tabular}{|c|c|c|}
\hline & Hombres \% & Mujeres \% \\
\hline Nahuatlismos & 39.12 & 36.90 \\
\hline
\end{tabular}

El cuadro VI presenta una distribución de términos entre los sexos. En él se puede observar que de manera general los varones son los que más usan estas voces indígenas, situación que es explicable debido a la mayor diversidad de actividades que tienen los hombres ya que la mujer se dedica más al hogar y a la educación de los hijos pequeños. Son pues los distintos ejercicios de los varones (empleo, viajes, etc.,) los que los motiva a usar más los indigenismos, a pesar de que - según mis estadísticas - las mujeres posean un porcentaje superior de nómina pasiva (solamente el $0.3 \%$ más). Si algunas voces caracterizarían a ambos sexos, éstas serían: nauyaca al masculino, y cenzontle al femenino.

Ahora bien, aparte de las variables sociolingüísticas utilizadas para caracterizar los diversos socilectos y medir así tanto la nómina pasiva como la activa de los nahuatlismos, lo que procede es observar la variación diafásica de este léxico. Éste suele aparecer en cualquier situación informal de la conversación e incluso en circunstancias más formales en tanto que el hablante no posea otro término: sea el caso de chicle, hule, náhuatl, quetzal, tomate, totopo y otros más. Su empleo no se restringe solamente al hogar, sino que es común oírlas en oficinas, escuelas, plazas y mercados a los que el hablante acude diariamente. Incluso, su audición es constante en algunos medios masivos de comunicación de carácter local, regional y nacional.

Como apunté antes, la presencia de términoos equivalentes hispánicos o de otra lengua afecta sobremanera la vitalidad de estos nahuatlismos en todos los sociolectos, sobre todo en el culto. Varios de ellos no han padecido desplazamiento alguno ante tal presencia y se mantienen firmes en su significado: aguacate, atole, cacahuate, chile, chocolate, elote, tomate, zapote y muchos más.

Otros, en cambio, se encuentran en franca competencia con voces hispánicas por un lado, o procedentes de otras o de la misma lengua indígena, por el otro. La adición hecha al cuestionario (específicamente la pregunta 5: ¿Con qué otro nombre conoce el objeto, fruta etc?), permite un acercamiento a estos términos equivalentes de la nómina pasiva de los hablantes. Habrá que realizar más adelante, con un método distinto al ahora empleado, investigaciones con el objeto de evaluar con qué porcentaje aparecen en la conversación tanto los nahuatlismos como los que no lo son.

A continuación anoto sólo los resultados que obtuve de los cuestionarios aplicados ${ }^{17}$ en los que se muestra que la voz nahua convive y a veces cede su uso al término equivalente, dependiendo de las circunstancias formal o informal de la comunicación.

17 Existirán diferencias obvias frente a los resultados que ofrece Lope Blanch (1979, específicamente 45-46 y 74 y ss.) debido a que en ambas investigaciones se emplearon métodos diferentes (me refiero a que en las encuestas que analiza Lope Blanch se tomaron en cuenta materiales de lengua escrita que yo no consideré, así como entre- 
Cuadro VII

\begin{tabular}{|c|c|c|c|}
\hline Nahuatlismo & Voz equivalente & Nahuatlismo & Voz equivalente \\
\hline Achichincle. & $\begin{array}{l}\text { Sirviente, gato, } \\
\text { compinche, } \\
\text { subordinado. }\end{array}$ & Achiote. & Recado rojo, recado. \\
\hline Ahulado. & Plástico. & Totopo. & Tostada. \\
\hline Matatena. & Yacses. & Apapachar. & Acariciar, chiquera, abrazar. \\
\hline Mecate. & Soga, cuerda, reata. & Cacahuate. & Maní \\
\hline Milpa. & $\begin{array}{l}\text { Parcela, siembra, } \\
\text { sembradio, siembros, } \\
\text { huerta. }\end{array}$ & Cempasúchil. & Flor de muertos \\
\hline Cuate. & Amigo, gemelo. & Chamaco. & Niño, chiquito, escuincle. \\
\hline Chapopote. & Pavimento. & Chapulín. & Grillo. \\
\hline Chicozapote. & Zapote. & Chicha. & Tetas, chuchús, pecho. \\
\hline Chicle. & Goma de mascar. & Chicloso. & Pegajoso. \\
\hline Chilpayate. & $\begin{array}{l}\text { Chiquito, niño, } \\
\text { escuincle, mocoso, } \\
\text { chamaco, bebé, nené. }\end{array}$ & Chipote. & Chuchuluco, chichón. \\
\hline Elote. & Maíz, mazorca.. & Chile. & Picante. \\
\hline Escuincle. & $\begin{array}{l}\text { Chiquito, niño, nené, } \\
\text { chilpayate, chamaco. }\end{array}$ & Huacal. & Caja. \\
\hline Guajolote. & Pavo, totol, cónono. & Huipil. & Terno. \\
\hline Jacal. & Choza. & Jitomate. & Tomate rojo. \\
\hline Petaca. & Maleta, bulto, nalga. & Tlapalería. & Ferretería. \\
\hline Mayate. & $\begin{array}{l}\text { Homosexual, } \\
\text { afeminado, } \\
\text { machacapiñas, } \\
\text { maricón. }\end{array}$ & Nauyaca. & Culebra, Cuatronarices. \\
\hline Mitotero. & Chismoso, mentiroso. & Zacate & Hierba. \\
\hline Nagual. & Brujo. & Tianguis. & Mercado. \\
\hline Nixtamal. & Masa, maíz cocido. & Tuza. & Topo. \\
\hline Paliacate. & Pañuelo. & Tecolote. & Búho. \\
\hline Papalote. & Papagayo, cometa. & Tlacuache. & Zorro. \\
\hline Petatearse. & Morirse. & & \\
\hline
\end{tabular}

vistas grabadas y no un cuestionario como el que yo utilicé) en zonas geográficas distintas. Añádase a esto que no todos los encuestados proporcionaron los sinónimos solicitados, y nunca se les preguntó si los usaban más o menos que los nahuatlismos, pregunta que, por lo demás, no hubiera arrojado datos lo suficientemente cercanos a la vitalidad de estas voces pues el hablante mismo se encuentra imposibilitado para evaluar el porcentaje de uso de una palabra o de su sinónimo equivalente. 


\section{Conclusiones}

La nómina pasiva general de los nahuatlismos en el español hablado en la frontera mexicana con Belice la conforman 109 voces y los grupos que más conocimiento tienen de ella son las mujeres, los miembros del grupo generacional III y las personas cultas.

En contraste, la mayor vitalidad de estas voces -el léxico activo- ${ }^{18}$ radica en los varones, la gente cuya edad oscila entre los 30 y los 50 años, y en la del nivel sociocultural medio.

Se trata de sustantivos concretos en su gran mayoría, que son usados para hacer referencia a la fauna y la flora de la propia región o de otras: (aguacate, chayote, zacate, tlacuache, coyote); la comida: (atole, chilaquiles, enchilada, mole, tamal, pozole, chirmole); los utensilios: (comal, hule, huacal, metate); el cuerpo humano: (chichi, chipote) y cualidades personales: (achichincle, cuate, chamaco, chilpayate, chiclero, escuincle, tocayo) principalmente.

Si bien los mayismos que aparecen con singular constancia en el habla de los chetumaleños deben su presencia a circunstancias históricas y geográficas específicas ${ }^{19}$, no se puede decir lo mismo de los nahuatlismos, pues varios de éstos son incorporaciones que se han dado en el español en general $\longrightarrow$ al menos en el español mexicano- desde antaño ${ }^{20}$ y no son - como los mayismos- de uso regional sino continental.

En suma, el barniz náhuatl que esmalta al español hablado en Chetumal —quizá menos que el que puede observarse en otros sitios de México- es considerable. El tiempo dirá qué tan fuertes son en su significación estas voces para conservarse dentro de la comunicación cotidiana de una sociedad urbana y globalizada cada vez mayor.

\section{Referencias bibliográficas}

Alba, O. (1976): "Indigenismos en el español hablado en Santiago", Anuario de letras 14, México, UNAM, págs. 71-100.

18 Cabe hacer mención que en las entrevistas realizadas con el objeto de realizar la descripción fonética y morfosintáctica del español de Chetumal, sólo documenté 9 nahuatlismos que equivalen al $0.02 \%$ del corpus léxico total: cuate ( 3 en 3 entrevistas), chamaco (13 en 10), chaquiste (1 vez), chicle ( 2 en 2), chiclero ( 1 vez), mole ( 1 $\mathrm{vez}$ ), tocayo (1 vez), zacate (1 vez), zopilote (1 vez). Si incluyo los nahuatlismos de uso general en México como aguacate, cacahuate, chile, chocolate, comal, nopal, pozole, tamal, zacate, etc., y algunos escuchados al margen de las entrevistas: pepenar, pinole, paliacate, toloache y otros, el porcentaje de este léxico vivo no variaría considerablemente $(0.10 \%$ aproximadamente).

19 La población chetumaleña tiene una ascendencia yucateca notoria. Esto hay que tomarlo en cuenta pues tanto las voces mayas como las nahuas llegaron a Chetumal con la población yucateca que conformaba la ciudad desde sus inicios. En 1904 -a sólo seis años de su fundación-, Payo Obispo (hoy Chetumal) contaba con 248 habitantes de los cuales la mayoría era proveniente de Belice y eran descendientes de los mestizos yucatecos y mayas que huyeron al sur del río Hondo durante la Guerra de Castas desarrollada en toda la zona durante la segunda mitad del siglo XIX.

20 A Yucatán llegaron los pochtecas a fines del siglo XV (Toscano: 1946), y a finales del XVI Alonso Ponce registra varios barrios de mexicanos en Mérida (Ramos: 2006) que seguramente sembraron sus voces autóctonas en el habla del pueblo. Por otro lado, se puede asegurar que los que llevaron las voces nahuas a Chetumal fueron seguramente los yucatecos avecindados en los distritos norteños de Belice (Corozal y Orange Walk) o los hijos de éstos quienes, invitados por el Almirante Othón Pompeyo Blanco, fundaron la ciudad en 1898, así como otros grupos sociales que llegaron del centro de México y de diversas zonas del país durante el poblamiento de la ciudad en las décadas sexta y séptima del siglo XX. 
Cabrera, L. (1988): Diccionario de aztequismos. México, Oasis.

Corominas, J. y J. Pascual (1980-1983): Diccionario Crítico-Etimológico Castellano e Hispánico. Madrid, Espasa-Calpe.

Henríquez Ureña, P. (1938): "Para la historia de los indigenismos", Buenos Aires, Biblioteca de Dialectología Hispánica III, Buenos Aires.

Henríquez Ureña, P. (1935): "Palabras antillanas en el Diccionario de la Academia" Revista de Filologia Hispánica XX y XXII, Buenos Aires, p. 175-186.

Lara, L. F. (1996): Diccionario del español usual de México. México, El Colegio de México.

Lope Blanch, J. M. (1979): Léxico indígena en el español de México. México, El Colegio de México. Malaret, A. (1946): Diccionario de americanismos. Buenos Aires, Emecé.

Montes, J. (1982): Dialectología general e hispanoamericana. Bogotá, Instituto Caro y Cuervo.

Morínigo, M. (1985): Diccionario manual de americanismos. Buenos Aires.

Neves, A. (1973): Diccionario de americanismos. Buenos Aires, Sopena.

Ramos, M. (2006): “Libros, ideas y educación al sureste de la Nueva España”. En Ramos, M. y C. Macías, (dirs.): El Caribe mexicano. Origen y conformación, siglos XVI y XVII. México, Miguel Ángel Porrúa y Universidad de Quintana Roo, págs. 329-423.

Real Academia Española (1970): Diccionario de la Real Academia Española. Madrid, Espasa-Calpe. Sala, M. et alli (1977): El léxico indigena del español americano. México y Bucarest,Academia Mexicana y Editora Academici Romane.

Santamaria, F. (1984): Diccionario de mexicanismos, México, Porrúa.

Simeon, R. (1977): Diccionario de la lengua náhuatl o mexicana. México, Siglo XXI.

Toscano, S. (1946): México prehispánico. México, Edit. E. Hurtado.

Vaquero, $\mathrm{M}^{\mathrm{a}}$. (1985): “El léxico indígena en el español hablado en Puerto Rico". En Léxico marítimo en Puerto Rico y otros estudios. Madrid, Playor, págs. 127-148. 